\title{
Molecular cloning and expression analysis of KIN10 and cold-acclimation related genes in wild banana 'Huanxi' (Musa itinerans)
}

\author{
Weihua Liu, Chunzhen Cheng, Gongti Lai, Yuling Lin and Zhongxiong Lai
}

\begin{abstract}
Banana cultivars may experience chilling or freezing injury in some of their cultivated regions, where wild banana can still grow very well. The clarification of the cold-resistant mechanism of wild banana is vital for cold-resistant banana breeding. In this study, the central stress integrator gene KIN1O and some cold-acclimation related genes (HOS1 and ICE1s) from the cold-resistant wild banana 'Huanxi' (Musa itinerans) were cloned and their expression patterns under different temperature treatments were analyzed. Thirteen full-length cDNA transcripts including 6 KIN10s, 1 HOS1 and 6 ICE1s were successfully cloned. Quantitative real-time PCR (qRT-PCR) results showed that all these genes had the highest expression levels at the critical temperature of banana $\left(13^{\circ} \mathrm{C}\right)$. Under chilling temperature $\left(4^{\circ} \mathrm{C}\right)$, the expression level of KIN1O reduced significantly but the expression of HOS1 was still higher than that at the optimal temperature $\left(28^{\circ} \mathrm{C}\right.$, control). Both KIN1O and HOS1 showed the lowest expression levels at $0^{\circ} \mathrm{C}$, the expression level of ICE1, however, was higher than control. As sucrose plays role in plant cold-acclimation and in regulation of KIN10 and HOS1 bioactivities, the sucrose contents of wild banana under different temperatures were detected. Results showed that the sucrose content increased as temperature lowered. Our result suggested that KIN10 may participate in cold stress response via regulating sucrose biosynthesis, which is helpful in regulating cold acclimation pathway in wild banana.
\end{abstract}

Keywords: Cold acclimation, Gene expression, KIN10, Musa itinerans, Sucrose

\section{Background}

Banana (Musa spp.) is one of the most important nutrient-rich crops, staple foods and ornamental plants cultivated in tropical and subtropical regions where temperature is relatively high. Nonetheless, considerable interests still exit in exploring banana cold-resistant genes and developing cold tolerant banana cultivars due to the chilling or freezing injuries they might experience at some of their cultivated regions (Yang et al. 2012). Up to now, however, no effective method has yet been developed to effectively solve the cold injury problem.

Wild banana germplasm resources are abundant in China, where various studies have been conducted over the past 20 years (Liu et al. 2007, 2012; Lai et al. 2007). Wild banana species are more cold resistant than

\footnotetext{
*Correspondence: laizx01@163.com

Institute of Horticultural Biotechnology, Fujian Agriculture and Forestry University, Fuzhou 350002, Fujian, China
}

cultivated ones and can grow under relatively lower temperatures (Lai et al. 2007). The discovery of beneficial wild banana gene resources is consequently of great usefulness for cold-resistance breeding of cultivated banana.

Cold acclimation can dramatically increase freezing tolerance of plants and is very important for extending their adaptation areas (Zhang et al. 2009). It was reported that sucrose can enhance cold hardening of plants by regulating expression of cold-acclimation-associated genes such as CBF (C-repeat/DRE-binding factor), ICE1 (inducer of CBF expression 1), HOS1 (high expression of osmotically responsive gene 1) and so on (Palonen and Junttila 2002; Rekarte-cowie et al. 2008). The expression of CBF1, was identified to be induced under cold stress (Lee et al. 2001). And its CBF AP2 motif combines with the CRT/ DRE element in the COR promoter to enhance COR expression and ultimately plant cold tolerance (Chen et al. 2014). In Arabidopsis, three CBF genes, CBF1, $C B F 2$, and $C B F 3$, were found (Stockinger et al. 1997; 
Gilmour et al. 1998; Medina et al. 1999) and their functions in cold acclimation were also identified (Novillo et al. 2007). ICE1 is a transcription factor that can activate the expression of CBFs, thus function in regulating the cold-induced transcriptome and freezing tolerance (Chinnusamy et al. 2003). HOS1, a functional E3 ligase targeting ICE1 for ubiquitination-mediated ICE1 degradation, is the negative regulator of plant cold responses and its expression is regulated by osmotic potent changes (Dong et al. 2006). Sucrose can significantly influence the osmotic potential of plant cells (Palonen and Junttila 2002). Interestingly, the bioactivity of SNF1-related protein kinase catalytic subunit alpha 10/11 (KIN10/11), the central integrator of transcription networks in plant stress and energy signaling (Baena-González et al. 2007), can be inhibited by high concentrations of sucrose (Thalor et al. 2012). KIN10/KIN11, in reverse, can phosphate the key enzyme of sucrose biosynthesis (sucrose phosphate synthase) (Thalor et al. 2012). These correlations suggest that KIN10/KIN11 should function in cold hardening of plants. Thus far, however, studies of banana KIN10/KIN11 and cold-acclimation related genes were rare due to the lack of sequence information.

Wild banana is widely distributed in all prefecture-level cities in Fujian Province, China (Lai et al. 2007). Among various germplasm resources, a wild banana population recently found in Huanxi, Fuzhou City, China, was found to be very tolerant to cold (Liu et al. 2012), making it very nice gene resources for cold-resistant genes and germplasm resources for cold-tolerant banana breeding. The release of Malaysian wild banana (Musa acuminata) genome data will be surely helpful for identification and characterization of these genes (D'Hont et al. 2012). So, in this study, KIN10, HOS1 and ICE1 (the target gene of $H O S 1)$ genes were identified by searching the Malaysian wild banana genome data and were cloned by RTPCR and/or RACE technologies from cold-resistant wild banana 'Huanxi' (Musa itinerans). Their expression patterns under different temperature, together with the sucrose content in leaves, were also analyzed. Our study could be helpful in understanding the cold acclimation responses of wild banana under different temperature and in exploring the function of KIN10 in cold response.

\section{Results}

In total, 6 KIN10 (KIN10-1-KIN10-6), 1 HOS1 and 6 ICE1 (ICE1-1-ICE1-6) genes were successfully cloned from cold-resistant wild banana 'Huanxi'. To the best of our known, this is the first report about cloning these genes from Musa spp. The generated sequences were submitted to GenBank, and the corresponding accession numbers were granted to KC127685, KC127686, KC127687, KC127688, KC127689, KC127690, JX678611,
KC157569, KC157570, KC157571, KC157572, KC157573 and $\mathrm{KC} 157574$, respectively.

Identification, characterization and bioinformatic analysis of KIN10 genes from cold-resistant wild banana 'Huanxi' Multiple-sequence BLAST search revealed that KIN1O1, KIN10-2 and KIN10-3 had similar ORF sequences that were $95.51 \%$ identical to the KIN10 of Malaysian wild banana (Musa acuminata) (GSMUA_Achr10 G09220_001). But the ORF sequences of KIN10-4, KIN10-5 and KIN10-6 shared lower identity (only $76.58 \%$ ). These sequence variations may be due to differences between genes or species. Bioinformatics prediction result revealed that all the 6 KIN1Os were basic, hydrophilic, and unstable proteins possessing transmembrane domains with predicted location in the nucleus or in membranes. Moreover, 21-26 phosphorylation sites were found in KIN10s (Table 1). Observed variations in the number and position of these phosphorylation sites suggest that some of their potential functions may be different. The KIN10s possessed 10-13 conserved domains, most of which were protein kinase domains (Additional file 1: Table S1). Phylogenetic analysis of KIN10 sequences generated the tree shown in Additional file 2: Figure S1. Besides the Malaysian wild banana KIN10, Phoenix dactylifera KIN10 and Elaeis guineensis KIN10 showed the closest relationship with wild banana 'Huanxi' KIN10s.

\section{Identification, characterization and bioinformatic analysis of HOS1 from cold-resistant wild banana 'Huanxi'}

The HOS1 cDNA was 2926 bp long and contained a 2904 bp ORF encoding 967 amino acids. Multiplesequence BLAST comparison showed HOS1 from 'Huanxi' shared high similarity $(93.95 \%)$ with the Malaysian wild banana HOS1 (GSMUA_Ach1G14640_001). The major difference between the two species was the presence of a $140 \mathrm{bp}$ insertion in the upstream region of the 'Huanxi'. On the basis of bioinformatics prediction analysis, HOS1 was shown to be a nuclear-localized, hydrophilic unstable protein without signal peptide. And 57 phosphorylation sites and a specific ELYS-like conserved domain were found in HOS1 (Table 1). Phylogenetic analysis of HOS1 sequences generated the tree shown in Additional file 3: Figure S3. Besides the Malaysian wild banana HOS1, Phoenix dactylifera HOS1 showed the closest relationship with wild banana 'Huanxi' HOS1.

Identification, characterization and bioinformatic analysis of ICE1 genes from cold-resistant wild banana 'Huanxi' Multiple-sequence BLAST search showed that the cloned ICE1-1-ICE1-4 genes shared higher identity 
Table 1 Information of KIN10s, HOS1 and ICE1s proteins in wild banana 'Huanxi'

\begin{tabular}{|c|c|c|c|c|}
\hline Protein name & Amino acid no. & Protein property & $\begin{array}{l}\text { Phosphorylation } \\
\text { site no. }\end{array}$ & $\begin{array}{l}\text { Predicted subcellular } \\
\text { location }\end{array}$ \\
\hline KIN10-1 & 491 & Hydrophilic, basic & 26 & Nucleus \\
\hline KIN10-2 & 513 & Hydrophilic, basic & 23 & Plasma membrane \\
\hline KIN10-3 & 513 & Hydrophilic, basic & 22 & Plasma membrane \\
\hline KIN10-4 & 506 & Hydrophilic, basic & 22 & Plasma membrane \\
\hline KIN10-5 & 513 & Hydrophilic, basic & 21 & Plasma membrane \\
\hline KIN10-6 & 513 & Hydrophilic, basic & 22 & Plasma membrane \\
\hline ICE1-1 & 547 & Hydrophilic, acidic & 23 & Nucleus \\
\hline ICE1-2 & 541 & Hydrophilic, acidic & 23 & Nucleus \\
\hline ICE1-3 & 542 & Hydrophilic, basic & 30 & Nucleus \\
\hline ICE1-4 & 536 & Hydrophilic, basic & 30 & Nucleus \\
\hline ICE1-5 & 503 & Hydrophilic, acidic & 20 & Nucleus \\
\hline ICE1-6 & 559 & Hydrophilic, acidic & 23 & Nucleus \\
\hline HOS1 & 967 & Hydrophilic, acidic & 57 & Nucleus \\
\hline
\end{tabular}

(97.52 \%) with Malaysian wild banana ICE1 (GSMUA_ Achr10 G18380_001) compared with ICE1-5 and ICE1-6 (92.08 \%). A 75 bp sequence, which was almost exactly the same size as that of ICE1 introns in Malaysian wild banana, was missing from the middle region of ICE1-1-ICE1-4 in wild banana 'Huanxi'. Other missing sequences in wild banana 'Huanxi' were a $16 \mathrm{bp}$ sequence absent from the upstream region of ICE1-3 and ICE1-4 and a 19 bp sequence deleted from the termination codon region of ICE1-1 and ICE1-3. ICE1-5 and ICE1-6 were 9 bp longer in wild banana 'Huanxi'. Interestingly, compared with the Malaysian wild banana ICE1, ICE1-5 of wild banana 'Huanxi' contained one more intron and one fewer exon and ICE1-6 possessed two additional introns, which might be results of alternative splicing in evolution (Keren et al. 2010). According to bioinformatics prediction, the first four wild banana 'Huanxi' ICE1s encoded similar numbers of amino acid residues, whereas the number of amino acid residues encoded by ICE1-5 and ICE1- 6 was quite different (Table 1). ICE1-1, ICE1-2, ICE1-5, and ICE1-6 were acidic proteins and ICE1-3 and ICE1-4 were basic. All six ICE1s were hydrophilic proteins with a specifically conserved basic-helix-loop-helix domain (IPR011598) as well as a non-integrated domain designated as PTH31945:SF0 and were predicted to be located in the nucleus. ICE5 and ICE6 featured an additional non-integrated conservative domain SSF55021 (Additional file 4: Table S2). The number of phosphorylation sites in ICE1s also varied from 20 to 30 among these 6 ICE1s (Table 1). ICE1s were predicted to be located in the nucleus and contained trans-membrane domains. Phylogenetic analysis of ICE1 sequences generated the tree shown in Additional file 5: Figure S2. Besides the Malaysian wild banana ICE1, the closest relationship was found between wild banana 'Huanxi' ICE1s and Elaeis guineensis ICE1.

\section{qRT-PCR analysis of KIN10, HOS1 and ICE1 genes in wild} banana under low-temperature stress

As shown in Fig. 1, the expression levels of KIN10, HOS1 and ICE1 genes differed significantly under different temperatures. At the banana critical temperature of $13{ }^{\circ} \mathrm{C}$, all these genes showed the highest expression levels. The expression levels of KIN10 decreased significantly at 4 and $0{ }^{\circ} \mathrm{C}$ compared with control. Although the up-regulation level decreased, the expression level of HOS1 at $4{ }^{\circ} \mathrm{C}$ was still higher than that of the control. At $0{ }^{\circ} \mathrm{C}$, however, the HOS1 expression level dropped to their lowest levels at $0{ }^{\circ} \mathrm{C}$, a temperature at which ICE1 expression levels were obviously increased.

\section{Sucrose contents in wild banana changed significantly under low-temperature stress}

Sucrose contents of samples extracted from plants subjected to $0,4,13$ and $28{ }^{\circ} \mathrm{C}$ for $36 \mathrm{~h}$ were shown in Fig. 1. Sucrose content rose markedly as the temperature dropped. Notably, the sucrose content in 'Huanxi' leaves at $4{ }^{\circ} \mathrm{C}$ was only about 1.16 -fold higher than control. At $0{ }^{\circ} \mathrm{C}$, however, the sucrose content was even 1.5 -fold higher than that of the control.

\section{Discussion}

Although banana cultivars grow in tropical and subtropical regions, they also suffer chilling or freezing damage at some part of its cultivated areas such as in Fujian, Guangdong, Guangxi and Yunnan provinces in China. Wild bananas are more tolerant to cold and thus are nice materials for exploring cold-resistant genes and clarifying the cold-acclimation mechanism. 


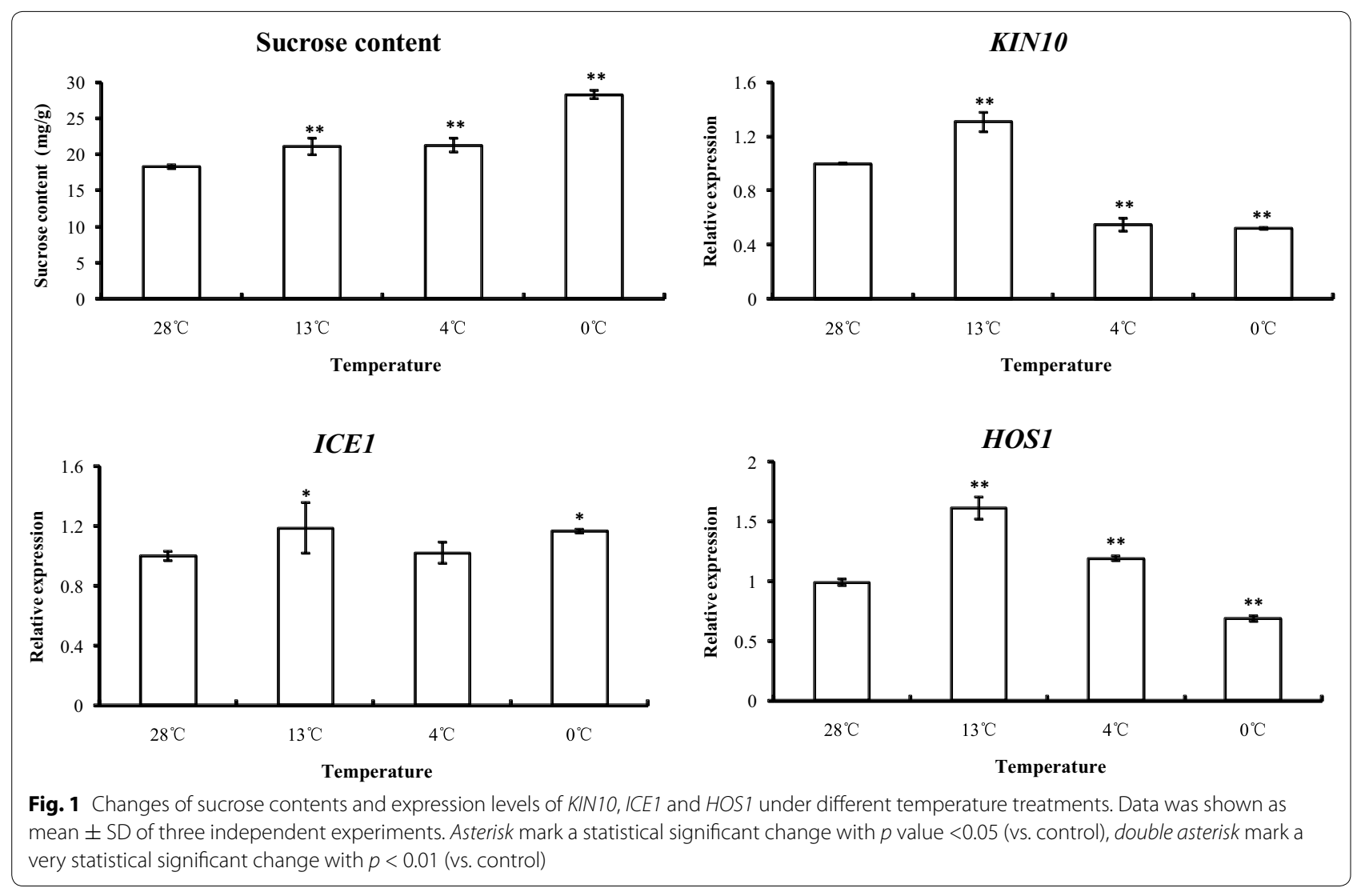

Totally, 6 KIN10s, 1 HOS1 and 6 ICE1s genes were successfully obtained. Sequence variations were found among KIN10 and ICE1 genes, which may be a consequence of evolutionary adaption to the environment: the Malaysian wild banana is distributed in tropical regions, whereas the wild banana 'Huanxi' is found at the northern margin of the southern subtropical region, and winter temperatures differ significantly between these two regions. Further research is needed to determine what, if any, functional differences are caused by the observed sequence differences.

Bioinformatic analysis also showed that all these genes are rich of phosphorylation sites, which suggest that phosphorylation may function a lot in plant cold resistance as phosphorylation was identified to play important role during the acquisition of freezing tolerance (Monroy et al. 1993; Komatsu et al. 1999; Schulze et al. 2012). Sequences analysis showed that protein-kinase domains of KIN10s were highly conserved, which might be the structural basis of the 'master molecular switch' in plant stress regulation (Baena-González et al. 2007). Observed variations in the number and position of these phosphorylation sites suggest that some of their potential functions may differ. The high number of conserved domains in wild banana KIN10s is unusual and reveals the diversity of these proteins' unique functions. At the same time, many of these conserved KIN10 domains are protein kinase domains, the structural basis of the 'master molecular switch' in plant stress regulation.

Notably, the evolutionary alternative splicing events were identified in ICE1 genes. As these genes are cold resistance-related functional genes from wild banana 'Huanxi', which is located in northern Fuzhou and often subject to low-temperature stress, we hypothesize that this alternative splicing phenomenon may be related to evolutionary adaptation to the environment to improve wild banana cold resistance. Whether any functional changes have occurred due to alternative splicing remains to be determined.

KIN10/KIN11 is an important regulator in various plant stress responses and its ability can be inhibited by high concentrations of sucrose (Baena-González et al. 2007; Thalor et al. 2012). The function of sucrose in plant cold acclimation (Palonen and Junttila 2002; Rekartecowie et al. 2008) indicated that KIN10/KIN11 might be also involved in plant cold acclimation. To prove this proposition, a new-found cold-resistant wild banana 'Huanxi' was used for cloning of the stress-related gene KIN1O and cold-acclimation related genes, HOS1 and ICE1. 
To compare their expression under different temperatures and to elucidate their function in cold-acclimation, expression levels of these genes at the optimal temperature $\left(28{ }^{\circ} \mathrm{C}\right)$, critical temperature $\left(13{ }^{\circ} \mathrm{C}\right)$, chilling temperature $\left(4{ }^{\circ} \mathrm{C}\right)$ and freezing temperature $\left(0{ }^{\circ} \mathrm{C}\right)$ were detected. Banana is basically a tropical crop growing at temperatures ranging from 13 to $38{ }^{\circ} \mathrm{C}$. Below $13{ }^{\circ} \mathrm{C}$ or above $38{ }^{\circ} \mathrm{C}$, banana growth ceases and $13{ }^{\circ} \mathrm{C}$ is thought to be the critical low temperature for banana growth (Nelson et al. 2006). Our study revealed that the relative expressions of KIN10s, HOS1 and ICE1s all reached the highest levels at the banana critical temperature. KIN10 could promote catabolic processes and suppress anabolic processes (Thalor et al. 2012), the up-regulation of KIN10, therefore, could be helpful in maintaining the energy balance and thus improve the cold resistance of wild banana at critical temperature. The expression of KIN10 was significantly suppressed at both 4 and $0{ }^{\circ} \mathrm{C}$. It was reported that once the cellular sucrose reached certain level, the activity of KIN10 would be suppressed (Baena-González et al. 2007; Thalor et al. 2012). At $4{ }^{\circ} \mathrm{C}$, the sucrose content in 'Huanxi' leaves was about $21.2 \mathrm{mg} / \mathrm{g}$ fresh weight, which might be the threshold for sucrose content of wild banana 'Huanxi'. KIN10 was also reported to be able to phosphorylate the key enzyme of sucrose biosynthesis, sucrose phosphate synthase (Thalor et al. 2012), so we inferred that KIN10 could participate in cold responses or cold-acclimation of wild banana through regulating sucrose biosynthesis.

The expression level of HOS1, an osmotically responsive gene, was still significantly higher than that of the control at $4{ }^{\circ} \mathrm{C}$, which indicated that non-freezing lowtemperature could induce the expression of HOS1. The sucrose content in leaves was higher at $4{ }^{\circ} \mathrm{C}$ than at $28{ }^{\circ} \mathrm{C}$, suggesting that the up-regulation of HOS1 may be a result of increasing osmotic potential in plant cells. HOS1 is a negative regulator of low temperature signal transduction that mediates the ubiquitination and degradation of ICE1 (Dong et al. 2006), which is a regulator of freezing tolerance in plants (Chinnusamy et al. 2003). At freezing temperature $\left(0{ }^{\circ} \mathrm{C}\right)$, expression of $\mathrm{HOS} 1$ was suppressed and expression of ICE1 was induced. The downregulation of low temperature negative regulator and up-regulation of freezing tolerance regulator reflected well the increase of cold tolerance.

\section{Conclusion}

In conclusion, we successfully cloned several genes of KIN10, HOS1 and ICE1 from cold-resistant wild banana 'Huanxi'. Expression analysis showed that expression of these genes and sucrose synthesis were significantly influenced by low temperature (Fig. 2): the expression of ICE1 was induced by low temperature; the expression of HOS1



was induced at non-freezing temperature but was suppressed at freezing temperature; the expression of KIN1O was influenced by low temperature and it might participate in cold-response by regulating sucrose biosynthesis. Our study could provide clues for improving banana cold resistance and for cold-tolerant banana breeding.

\section{Methods}

\section{Plant materials and treatments}

Plants of cold-resistant wild banana 'Huanxi' (Musa itinerans) were obtained from the Wild Banana Germplasm Nursery of the Institute of Horticultural Biotechnology, Fujian Agriculture and Forestry University, Fuzhou, China. For temperature treatments, 25-day-old plantlets growing at $28^{\circ} \mathrm{C}$ were subjected to 0,4 and $13^{\circ} \mathrm{C}$ for $36 \mathrm{~h}$ with plantlets growing at $28^{\circ} \mathrm{C}$ as control.

\section{Gene cloning and sequence analysis}

Homologous gene sequences in GenBank database (http://www.ncbi.nlm.nih.gov) and in the Malaysian wild banana (Musa acuminata) genome-wide data (http:// banana-genome.cirad.fr) were downloaded and used as reference sequences for determination of the conserved regions and for primer design. Information of all the used primers was listed Additional file 6: Table S3. The gene cloning processes were shown in Additional file 7: Figure S4. The obtained cDNA sequences were all submitted to the GenBank database (http://www.ncbi.nlm.nih.gov).

Bioinformatics analysis of these obtained genes and their deduced protein sequences were performed according to Tian et al. (2015). Amino acid sequences of KIN10s, ICE1s, and HOS1 were deduced and analyzed with the ExPASy Protparam tool (Wilkins et al. 1999). BLAST 
searches were performed using the NCBI server. Multiple alignment and structural analysis of deduced proteins was performed using DNAMAN 6.0. Signal peptide and protein subcellular localization information were predicted using SignalP4.0 Server (Petersen et al. 2011) and PSORT (Nakai 2000) software, respectively. Protein transmembrane regions and orientations were predicted using the Tmpred program. Conserved domain fields were identified with InterProScan (http://www.ebi.ac.uk/interpro/ scan.html) (Mitchell et al. 2015) and phosphorylation sites were predicted using the NetPhos 2.0 Server (http://www. cbs.dtu.dk/services/NetPhos/) (Wong et al. 2007). Phylogenetic analyses were performed using neighbor-joining in MEGA 5.02 (Tamura et al. 2011).

\section{qRT-PCR expression analysis of KIN10s, ICE1s and HOS1}

To explore the relationship of these cold-resistance genes and the cold stress response mechanism in 'Huanxi' wild banana under low-temperature stress, we conducted a gene expression analysis using qRT-PCR. After low temperature treatment, leaves were sampled for total RNA extraction. qRT-PCR analyses were performed as described by Lin and Lai (2013) on Roche LightCycler480 (Roache, Basel, Switzerland) with $18 S$ rRNA as the internal control. Conserved regions of KIN1Os, HOS1 and ICE1-1-ICE1-4 were used as templates for primer design. Three biological and technical replicates were made for each treatment. Primers used were listed in Additional file 8: Table S4.

\section{Sucrose content determination}

The same extracts obtained from leaves exposed to 0,4 , 13 and $28{ }^{\circ} \mathrm{C}$ (control) for $36 \mathrm{~h}$ and used for the qPCR analysis were analyzed for sucrose content. The sucrose contents in leaves were measured by using the method of (Xue 1985).

\section{Additional files}

Additional file 1: Table S1. Conserved domains in KIN10s of wild banana'Huanxi'.

Addtional file 2: Figure S1. Phylogenetic tree of the amino acid sequences of KIN10.

Addtional file 3: Figure S2. Phylogenetic tree of the amino acid sequences of HOS1.

Additional file 4: Table S2. Conserved domains in ICE1s of wild banana 'Huanxi'.

Addtional file 5: Figure S3. Phylogenetic tree of the amino acid sequences of ICE1.

Additional file 6: Table S3. Information of primers used for cloning of KIN10s, HOS1 and ICE1s from wild banana 'Huanxi'.

Addtional file 7: Figure S4. Cloning process of the cold-acclimation related genes in wild banana 'Huanxi'.

Additional file 8: Table S4. Information of primers used for qRT-PCR.

\section{Abbreviations}

PCR: Polymerase chain reaction; KIN10s: SNF1-related protein kinase catalytic subunit alpha 10; HOS1: High expression of osmotic stress-regulated gene expression 1; CBF: CRT(C-repeat/dehydration response element)/ DRE(dehydration responsive element)-binding factor; ICE1: Inducer of CBF expression 1; RT-PCR: Reverse transcription PCR; qRT-PCR: Quantitative realtime PCR; RACE: Rapid amplification of CDNA ends; DNA: Deoxyribonucleic acid; RNA: Ribonucleic acid; CDNA: Complementary DNA; bp: Base pair; OFR: Open reading frame; CDS: Coding sequence; UTR: Untranslated region.

\section{Authors' contributions}

WL, ZL, CC and YL conceived and designed the experiments. WL and GL performed the experiments. $\mathrm{WL}, \mathrm{CC}$ and $\mathrm{ZL}$ analyzed the data. $\mathrm{WL}, \mathrm{CC}$ and $\mathrm{ZL}$ wrote the paper. All authors read and approved the final manuscript.

\section{Acknowledgements}

This work was supported by the earmarked Fund for China Agriculture Research System (CARS-32-11) and the Fujian Provincial Key Science and Technology Project (2015NZ0002-1, 2008N2001).

\section{Competing interests}

The authors declare that they have no competing interests.

Received: 2 November 2015 Accepted: 13 December 2015

Published online: 30 December 2015

\section{References}

Baena-González E, Rolland F, Thevelein JM, Sheen J (2007) A central integrator of transcription networks in plant stress and energy signaling. Nature 448:938-943

Chen J, Tian Q, Pang T, Jiang L, Wu R, Xia X, Yin W (2014) Deep-sequencing transcriptome analysis of low temperature perception in a desert tree, Populus euphratica. BMC Genom 15:326

Chinnusamy V, Ohta M, Kanrar S, Lee BH, Hong X, Agarwal M, Zhu JK (2003) ICE1: a regulator of cold-induced transcriptome and freezing tolerance in Arabidopsis. Gene Dev 17:1043-1054

Dong CH, Agarwal M, Zhang Y, Xie Q, Zhu JK (2006) The negative regulator of plant cold responses, HOS1, is a RING E3 ligase that mediates the ubiquitination and degradation of ICE1. P Natl Acad Sci USA 103:8281-8286

D'Hont A, Denoeud F, Aury J, Baurens F, Carreel F, Garsmeur O, Noel B, Bocs S, Droc G, Rouard M, Silva CD, Jabbari K, Cardi C, Poulain J, Souquet M, Labadie K, Jourda C, Lengellé J, Rodier-Goud M, Alberti A, Bernard M, Correa M, Ayyampalayam S, Michael MR, Leebens-Mack J, Burgess D, Freeling M, Mbéguié-A-Mbéguié D, Chabannes M, Wicker T, Panaud O, Barbosa J, Hribova E, Heslop-Harrison P, Habas R, Rivallan R, Francois P, Poiron C, Kilian A, Burthia D, Jenny C, Bakry F, Brown S, Guignon V, Kema G, Dita M, Waalwijk C, Joseph S, Dievart A, Jaillon O, Leclercq J, Argout X, Lyons E, Almeida A, Jeridi M, Dolezel J, Roux N, Risterucci A, Weissenbach J, Ruiz M, Glaszmann J, Quétier F, Yahiaoui N, Wincker P (2012) The banana (Musa acuminata) genome and the evolution of monocotyledonous plants. Nature 488:213-219

Feller A, Hernandez JM, Grotewold E (2006) An ACT-like domain participates in the dimerization of several plant basic-helix-loop-helix transcription factors. J Biol Chem 281:28964-28974

Gilmour SJ, Zarka DG, Stockinger EJ, Salazar MP, Houghton JM, Thomashow MF (1998) Low temperature regulation of the Arabidopsis CBF family of AP2 transcriptional activator as an early step in cold-induced COR gene expression. Plant J 16:433-442

Keren H, Lev-Maor G, Ast G (2010) Alternative splicing and evolution: diversification, exon definition and function. Nat Rev Genet 11:345-355

Komatsu S, Karibe H, Hamada T, Rakwal R (1999) Phosphorylation upon cold stress in rice (Oryza sativa L.) seedlings. Theor Appl Genet 98:1304-1310

Lai ZX, Chen Y, Lin YL, Zhao QY, Chen YT (2007) Discovery and taxonomy of wild banana (Musa spp.) in Fuzhou. Subtrop Agric Res 3:1-5 (in chinese)

Lee H, Xiong L, Gong Z, Ishitani M, Stevenson B, Zhu JK (2001) The Arabidopsis HOS1 gene negatively regulates cold signal transduction and encodes a RING-finger protein that displays cold-regulated nucleo-cytoplasmic partitioning. Gene Dev 15:912-924 
Lin YL, Lai ZX (2013) Superoxide dismutase multigene family in longan somatic embryos: a comparison of CuZn-SOD, Fe-SOD, and Mn-SOD gene structure, splicing, phylogeny, and expression. Mol Breed 32:595-615

Liu DB, Wei JY, Li SP, Peng M (2007) Cloning and characterization of a fulllength cDNA encoding the RuBPCase small subunit in banana. Chin J Trop Crops 28:57-61 (in chinese)

Liu WH, Lai ZX, Lai GT,Lin YL, Liu SC et al (2012) A study on taxonomic status and in vitro propagation of the wild banana (Musa spp.) from Huanxi Town in Fujian Province of China. In: 5th International Symposium on Tropical and Subtropical Fruits. ISHS, Guangzhou

Medina J, Bargues M, Terol J, Pérez-Alonso M, Salinas J (1999) The Arabidopsis CBF gene family is composed of three genes encodes AP2 domain-containing proteins whose expression is regulated by low temperature but not by abscisic acid or dehydration. Plant Physiol 119:463-470

Mitchell A, Chang HY, Daugherty L, Fraser M, Hunter S, Lopez R, McAnulla C, McMenamin C, Nuka G, Pesseat S, Sangrador-Vegas A, Scheremetjew M, Rato C, Yong SY, Bateman A, Punta M, Attwood TK, Sigrist CJA, Redaschi N, Rivoire C, Xenarios I, Kahn D, Guyot D, Bork P, Letunic I, Gough J, Oates M, Haft D, Huang HZ, Natale DA, Wu CH, Orengo C, Sillitoe I, Mi HY, Thomas PD, Finn RD (2015) The InterPro protein families database: the classification resource after 15 years. Nucleic Acids Res 43:213-221

Monroy AF, Sarhan F, Dhindsa RS (1993) Cold-induced changes in freezing tolerance, protein phosphorylation, and gene expression (evidence for a role of calcium). Plant Physiol 102:1227-1235

Nakai K (2000) Protein sorting signals and prediction of subcellular localization. Adv Protein Chem 54:277-344

Nelson SC, Ploetz RC, Kepler AK (2006) Musa species (banana and plantain). Species profiles for pacific island agro forestry 2006:9-22

Novillo F, Medina J, Salinas J (2007) Arabidopsis CBF1 and CBF3 have a different function than CBF2 in cold acclimation and define different gene classes in the CBF regulon. Proc Natl Acad Sci USA 104:21002-21007

Palonen P, Junttila O (2002) Carbohydrates and winter hardness in red raspberry. Acta Horticulturae 585:573-577

Petersen TN, Brunak S, Heijne GV, Nielsen H (2011) SignalP 4.0: discriminating signal peptides from transmembrane regions. Nat Methods 8:785-786

Rekarte-cowie I, Omar S, Ebshish OS, Mohamed KS, Pearce RS (2008) Sucrose helps regulate cold acclimation of Arabidopsis thaliana. J Exp Bot 59:4205-4217

Schulze WX, Schneider T, Starck S, Martinoia E, Trentmann O (2012) Cold acclimation induces changes in Arabidopsis tonoplast protein abundance and activity and alters phosphorylation of tonoplast monosaccharide transporters. Plant J 69:529-541

Stockinger EJ, Gilmour SJ, Thomashow MF (1997) Arabidopsis thaliana CBF1encodes an AP2 domain-containing transcriptional activator that binds to the C-repeat/DRE, acis-acting DNA regulatory element that stimulates transcription in response to low temperature and water deficit. Proc Natl Acad Sci USA 94:1035-1040

Tamura K, Peterson D, Peterson N, Stecher G, Nei M, Kumar S (2011) MEGA5: molecular evolutionary genetics analysis using maximum likelihood, evolutionary distance, and maximum parsimony methods. Mol Biol Evol 28:2731-2739

Thalor SK, Berberich T, Lee SS, Yang SH, Zhu XJ, Imai R, Takahashi Y, Kusano $T$ (2012) Deregulation of sucrose-controlled translation of a bZIP-type transcription factor results in sucrose accumulation in leaves. PLoS One 7:e33111

Tian QL, Lin YL, Yang MM, Zhang DM, Lai RL, Lai ZX (2015) DIRan3A is involved in hormone, light, and abiotic stress responses in embryogenic callus of Dimocarpus longan Lour. Gene 569:267-275

Wilkins MR, Gasteiger E, Bairoch A, Sanchez JC, Williams KL, Appel RD, Hochstrasser DF (1999) Protein identification and analysis tools in the ExPASy server. Methods Mol Biol 112:531-552

Wong YH, Lee TY, Liang HK, Huang CM, Wang TY, Yang YH, Chu CH, Huang HD, Ko MT, Hwang JK (2007) KinasePhos 2.0: a web server for identifying protein kinase-specific phosphorylation sites based on sequences and coupling patterns. Nucleic Acids Res 35:W588-W594

Xue YL (1985) Plant physiology experiment. Shanghai Science and Technology Press, Shanghai, pp 135-138 (in chinese)

Yang QS, Wu JH, Li CY, Wei YR, Sheng O, Hu CH, Kuang RB, Huang YH, Peng XX McCardle JA, Chen W, Yang Y, Rose JKC, Zhang S, Yi GJ (2012) Quantitative proteomic analysis reveals that antioxidation mechanisms contribute to cold tolerance in plantain (Musa paradisiaca L., ABB Group) seedlings. Mol Cell Proteomics 30:1853-1869

Zeng XB, Li FN, Xu LB, Yang H, Lin ZX (1989) Investigation of the wild banana in Guangdong. Acta Horticulturae Sinica 16:95-100 (in chinese)

Zhang CZ, Fei SZ, Warnke S, Li LJ, Hannapel D (2009) Identification of genes associated with cold acclimation in perennial ryegrass. J Plant Physiol 166:1436-1445

\section{Submit your manuscript to a SpringerOpen ${ }^{\odot}$ journal and benefit from:}

- Convenient online submission

- Rigorous peer review

- Immediate publication on acceptance

- Open access: articles freely available online

- High visibility within the field

- Retaining the copyright to your article

Submit your next manuscript at $>$ springeropen.com 